\title{
The \begin{tabular}{c} 
Oncologist \\
\hline Sarcomas \\
\hline
\end{tabular}
}

\section{Understanding Micrometastatic Disease and Anoikis Resistance in Ewing Family of Tumors and Osteosarcoma}

\author{
Sandra J. Strauss, ${ }^{\text {a }}$ Tony Ng, ${ }^{\text {b }}$ Ariadna Mendoza-Naranjo, ${ }^{\text {a Jeremy Whelan, }},{ }^{a}$ \\ POUL H.B. SORENSEN ${ }^{b}$ \\ ${ }^{\mathrm{a} U C L}$ Cancer Institute, London, United Kingdom; ${ }^{\mathrm{b}}$ Department of Molecular Oncology, British Columbia \\ Cancer Research Centre, Vancouver, British Columbia, Canada
}

Key Words. Micrometastatic disease • Anoikis • Osteosarcoma • Ewing family of tumors • EWS-FLI1

\begin{abstract}
Disclosures
Sandra J. Strauss: None; Tony Ng: None; Ariadna Mendoza-Naranjo: None; Jeremy Whelan: None; Poul H.B. Sorensen: None.
\end{abstract}

Section editor Laurence Baker discloses that he chairs the career development committee of Sarcoma Alliance for Research through Collaboration (SARC) and has, as a faculty member of the University of Michigan, a conflict of interest management plan for his SARC activities. Section editor Jaap Verweij has disclosed no financial relationships relevant to the content of this article.

The content of this article has been reviewed by independent peer reviewers to ensure that it is balanced, objective, and free from commercial bias.

\section{LEARNING OBJECTIVES}

After completing this course, the reader will be able to:

1. Explain the importance of resistance to anoikis in the development of metastases.

2. Describe the mechanisms of anoikis resistance in EFTs and osteosarcoma and their potential use in development of new therapies.

\section{GME} This article is available for continuing medical education credit at CME.TheOncologist.com.

\section{AbstraCT}

Detection of micrometastatic tumor cells in the bone marrow or peripheral blood of patients with Ewing family of tumors (EFTs) and osteosarcoma has been shown to correlate with poor outcome. Although one of the aims of chemotherapy is eradication of micrometastatic disease, these cells vary phenotypically from primary tumor cells and appear to be more resistant to chemotherapy. As a barrier to metastasis, cells normally undergo a form of cell death termed anoikis after they lose contact with the extracellular matrix or neighboring cells. Tumor cells that acquire malignant poten- tial have developed mechanisms to resist anoikis and thereby survive after detachment from their primary site and while traveling through the circulation. Investigating mechanisms of resistance to anoikis, therefore, provides a valuable model to investigate regulation of micrometastatic disease. This review focuses on the current understanding of the mechanisms involved in mediating cell survival and resistance to anoikis in EFTs and osteosarcoma and discusses future studies that may help to identify novel therapeutics targeted at micrometastatic disease. The Oncologist 2010;15:627-635

Correspondence: Sandra J. Strauss, M.D., Ph.D., UCL Cancer Institute, Paul O'Gorman Building, 72 Huntley Street, London WC1E 6BT, U.K. Telephone: 44-207-380-9358; Fax: 44-207-380-9055; e-mail: s.strauss@ ucl.ac.uk Received March 30, 2010; accepted for publication April 13; first published online in The Oncologist Express on May 17, 2010. CAlphaMed Press 1083-7159/2010/\$30.00/0 doi: 10.1634/theoncologist.2010-0093 


\section{INTRODUCTION}

The development of distant metastases is the main cause of death in patients with malignant disease [1]. Micrometastatic disease refers to the presence of clinically undetectable tumor cells at diagnosis, or cells that remain present after treatment to eradicate the primary tumor. These circulating tumor cells (CTCs), found in the peripheral blood of cancer patients, and disseminated tumor cells (DTCs), which are distributed by blood circulation and found in the bone marrow, are purported to be the origin of metastatic disease, and one of the aims of chemotherapy is their elimination. Several clinical studies in solid tumors have shown that detection of micrometastatic disease, either in the bone marrow or peripheral blood, is accompanied by a substantially worse prognosis [2]. The most robust data come from a study of $>4,000$ patients with breast cancer, in which tumor cells in the bone marrow predicted the development of skeletal and other metastases [3]. CTCs and DTCs were found to be resistant to chemotherapy in a subset of patients, and their presence after adjuvant therapy is associated with an extremely poor prognosis [4-6]. Several studies performed to characterize the phenotype of DTCs have demonstrated a lack of expression of the proliferationassociated antigen Ki67. Such data may explain the resistance of DTCs to chemotherapy, and studies examining the role of further noncytotoxic adjuvant therapy in these patients are ongoing [7]. Not all patients with micrometastatic disease develop metastases, however, and the biology of micrometastatic cells remains poorly understood. Animal models indicate that a significant fraction of these cells may die or remain in a dormant state, and it is ill understood what conditions are required for escape from the dormant phase into the active phase of metastasis development [8]. The long latency period that occurs in some patients between initial treatment and clinical evidence of recurrence is attributed to this tumor cell dormancy.

Osteosarcoma and Ewing family of tumors (EFTs) are two of the most common malignancies arising in adolescents and young adults. Osteosarcoma is a mesenchymal tumor of the bone that can arise in any location but is most commonly seen in the long bones of the leg. The majority of patients present with localized disease, but with surgery alone, $80 \%-90 \%$ will die from their disease, generally as a result of the development of lung metastases [9]. The use of multiagent chemotherapy has substantially improved the outcome of these patients, with 5-year survival rates now reaching approximately $60 \%-70 \%[10,11]$. However, many patients still die because of the development of metastases, despite good local control, suggesting that micro- metastases are not being eradicated by the current treatment approaches in all patients and, thus, lead to distant recurrences. The presence of micrometastases in patients with osteosarcoma was demonstrated in a study by Bruland and colleagues, who used two antibodies recognizing osteosarcoma-associated antigens to immunomagnetically isolate tumor cells from the bone marrow and peripheral blood of patients. They found that 15 of $28(54 \%)$ patients without overt metastases at diagnosis had malignant cells in the bone marrow, and the presence of these tumor cells correlated with disease relapse and poor outcome [12].

The EFTs comprise Ewing sarcomas arising from bone and extraosseous Ewing sarcoma arising from soft tissues (which includes peripheral neuroectodermal tumors). The EFTs are characterized by the presence of specific chromosomal translocations that fuse the $5^{\prime}$ portion of the $22 \mathrm{q} 12$ $E W S$ gene with $3^{\prime}$ sequences of different ETS family genes [13]. Among the observed fusion genes, EWS-FLII is present in nearly $85 \%$ of EFTs whereas the $E W S-E R G$ gene occurs in the majority of the remaining $15 \%$ of EFTs $[14,15]$. Like osteosarcoma, EFTs are considered to be systemic malignancies, and major improvements in outcome have been made with the use of multidrug chemotherapy such that 5-year survival rate now approaches $70 \%$ for patients with localized disease at diagnosis $[16,17]$. Patients most commonly die as a result of the development of lung metastases, but the bone and bone marrow may also be involved [18]. In a study of 172 patients, micrometastases were found in the bone marrow or peripheral blood in approximately $20 \%$ of those with localized disease at diagnosis when examined by reverse transcription-polymerase chain reaction for the EWS-FL1 or EWS-ERG rearrangement [19]. In that study, the presence of micrometastases again correlated with a higher risk for relapse, and the outcome was comparable with that of patients with known metastases.

\section{Regulation of Metastasis}

Metastasis of cancer cells to distant sites is a multistep process that involves the cancer cells becoming dissociated from the primary site, intravasating into and surviving in the circulation, and extravasating and growing in a distant target organ (Fig. 1). At a cellular level, micrometastatic cells are thought to arise through detachment from the primary tumor after acquiring an invasive phenotype that allows them to migrate through the basement membrane. Several genes have been shown to be involved in the promotion of this invasive phenotype and initiation of metastasis, including Snail, Twist, and, more recently, YB-1, 


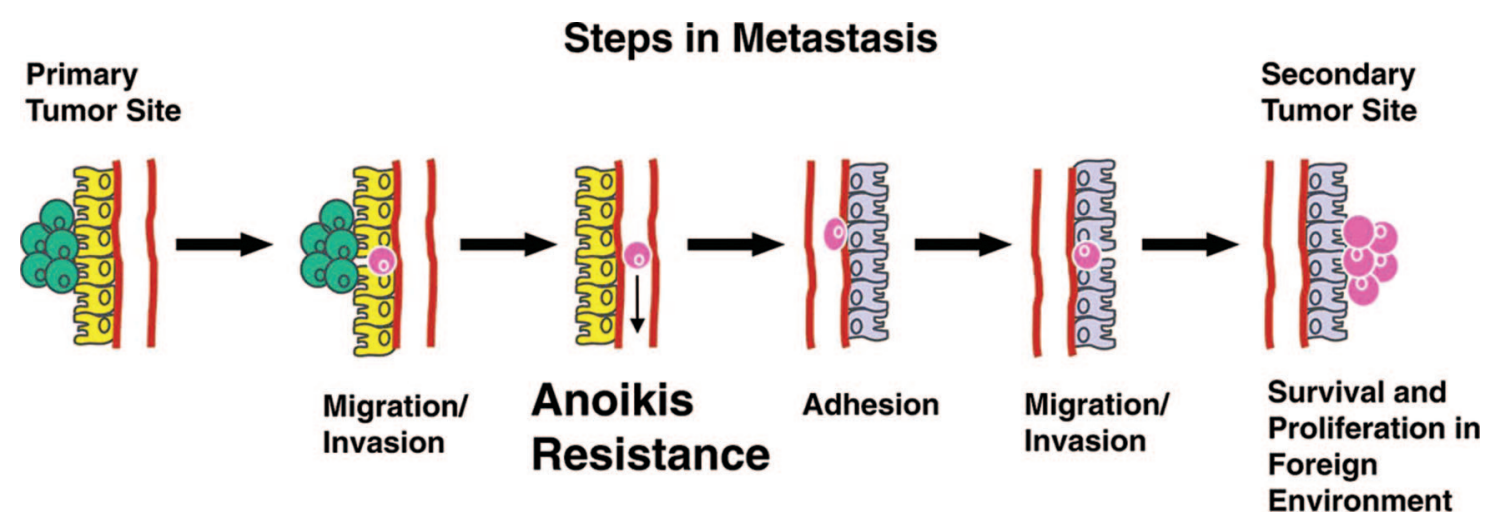

Figure 1. Metastasis involves several steps in which tumor cells disseminate from the primary site to secondary metastatic sites. Anoikis resistance allows tumor cells to survive in the absence of adhesion to the extracellular matrix, in the circulation or lymphatic system and travel to secondary sites in distant organs. Adapted from Simpson CD, Anyiwe K, Schimmer AD. Anoikis resistance and tumor metastasis. Cancer Lett 2008;272:177-185; with permission from Elsevier.

which act together to allow loss of cell adhesion and increased cell migration [20-22]. Once cells have entered into the circulation, they lack proper extracellular matrix (ECM) attachment, which in normal nonhematopoietic cells results in a special form of cell death called anoikis (a Greek word meaning homelessness) [23]. The initiation and execution of anoikis is mediated by caspase-dependent apoptosis, and its role is likely to be preventing ectopic growth of cells at inappropriate body sites [24]. In order to metastasize to distant sites, tumor cells must acquire the ability to resist anoikis and survive in the absence of adhesion to the ECM. This review focuses on the current knowledge of mechanisms of resistance to anoikis in malignant cells, and specifically in EFTs and osteosarcoma in an effort to increase our understanding of the biology of micrometastatic disease in these tumors.

\section{Anoikis Resistance DURing Metastasis}

Anoikis has critical physiological roles by regulating cell homeostasis in tissues, and it is also important during development, in tissue remodeling, and in the resolution of wound-healing responses [25-27]. In contrast, transformed cells and most cell lines established from human tumors show anoikis resistance when grown under nonadherent (anchorage-independent) culture conditions, surviving despite the absence of a matrix. Consistent with this, transformation by various oncogenes including those encoding activated phosphoinositide 3-kinase (PI3K), its downstream effector Akt, and activated focal adhesion kinase (FAK), as well as loss of tumor suppressor genes, can render cells resistant to anoikis and promote anchorage-independent growth [24, 28-33].

Resistance to anoikis has been shown to significantly contribute to the malignancy of different cancers, and protection from anoikis has been proven to facilitate both the survival and expansion of metastatic cells [29, 33]. Using a genomewide functional screening approach, Douma and coworkers looked for genes that enable cells to grow in anchorage-independent conditions and identified $\operatorname{TrkB}$ as conferring anoikis resistance [33]. TrkB, a receptor tyrosine kinase that regulates essential neuronal processes, including neuronal survival, was found to protect nonmalignant epithelial cells against anoikis by a mechanism that requires activation of the PI3K/Akt pathway, while completely failing to rescue programmed cell death by other proapoptotic insults, indicating that TrkB does not act as a general prosurvival factor [33]. A further role in tumorigenesis and metastasis was confirmed after i.v. injection of epithelial cells overexpressing TrkB into immunodeficient mice, which then demonstrated an aggressive metastatic capacity in vivo, suggesting that loss of anoikis may be sufficient to endow cells with metastatic capacity [33]. Recently, a direct correlation between anoikis resistance and metastatic capacity was demonstrated [34]. Using a loss-of-function screening approach, Cheng and colleagues identified the LKB1-dependent kinase SIK1 as a key modulator of anoikis. They showed that silencing SIK1 in tumor cells with short hairpin RNAs decreased p53 expression and phosphorylation under anchorage-independent conditions and contributed to the development of micrometastases in the absence of primary tumors in immunodeficient mice [34].

A range of proteins has been found to regulate complex signaling pathways directly associated with the suppression of anoikis in transformed epithelial cell models. Beside the well-known involvement of PI3K/Akt, other protein kinases have also been implicated as central regulators in detachment-independent growth in tumor cells, including Src, integrin-linked kinase (ILK), extracellular signal-related kinase (ERK), and TrkA [35-39]. A role has also been 
shown for signal transducer and activator of transcription 3 , whose activation is important for oncogenic insulin-like growth factor-1 receptor (IGF-1R)-induced anchorageindependent growth [40]. Additionally, Rho GTPases, which control many aspects of intracellular actin dynamics and can be regulated by integrin signaling, have been linked to anoikis resistance through activation of the ERK, c-Jun $\mathrm{N}$-terminal kinase, and p38 mitogen-activated protein kinase (MAPK) pathways [41].

\section{ANOIKIS RESISTANCE IN EFTS}

The molecular mechanisms underlying the progression of EFTs and the development of metastases, as well as the mechanisms governing anoikis resistance, are only beginning to be elucidated (Table 1). Lawlor and colleagues demonstrated that EFT cell lines readily survive in anchorage-independent conditions, persisting as multicellular spheroid clusters [42]. These spheroids show considerable phenotypic differences from their monolayer counterparts, including dramatically reduced proliferation with associated suppression of cyclin D1 levels, greater dependence on serum stimulation, and hyperactivation of various oncogenic signaling pathways including the PI3K/Akt and RasERK pathways. Moreover, the ability of EFT cell lines to proliferate under such conditions is particularly dependent on activation of Akt, because chemical inhibition of Akt further reduces cyclin D1 levels in EFT spheroids. This is in line with numerous studies in epithelial cancers demonstrating the importance of sustained PI3K/Akt pathway activation in anoikis resistance [43-45]. In EFTs, PI3K/Aktmediated cell growth and survival has been demonstrated to occur via activation of IGF-1R signaling, which is also required for maintaining the malignant behavior of these tumors [46-48]. IGF-1R-mediated suppression of anoikis through PI3K/Akt activation has been demonstrated in transformed mesenchymal cell models and in EFT cell lines, in which antisense oligonucleotides targeting IGF-1R lead to a decrease in cell proliferation, as well as an increase in anoikis-induced apoptosis [49-51]. Preclinical models confirmed the antitumor activity of IGF-1R inhibitors, and monoclonal IGF-1R antibodies and small molecule inhibitors are currently in clinical development, with encouraging efficacy observed in phase I/II trials [50-53]. Inhibition of the IGF-1R pathway appears to be a good therapeutic target for treatment of primary disease, but it may also be of benefit for treatment of micrometastases.

EFT spheroids show more highly developed cell-cell junctions than monolayer cultures, suggesting that anoikis resistance in such anchorage-independent conditions might also depend on these cell-cell interactions. To explore this phenomenon further, Kang et al. [54] investigated the role
Table 1. Cell characteristics, molecules, and pathways involved in conferring resistance to anoikis in EFT and osteosarcoma

\begin{tabular}{|c|c|}
\hline $\begin{array}{l}\text { Cell characteristics, molecules, and } \\
\text { pathways }\end{array}$ & References \\
\hline \multicolumn{2}{|l|}{ EFT } \\
\hline $\begin{array}{l}\text { Reduced cell proliferation with } \\
\text { suppression of cyclin-D }\end{array}$ & {$[42,54]$} \\
\hline Activation of PI3K/Akt signaling pathway & {$[42,54]$} \\
\hline Activation of IGF-1R signaling pathway & {$[50]$} \\
\hline $\begin{array}{l}\text { Formation of multicellular spheroids with } \\
\text { increased cell-cell contacts resulting from } \\
\text { increased E-cadherin expression }\end{array}$ & {$[54]$} \\
\hline Increased ErbB-4 expression & [54] \\
\hline \multicolumn{2}{|l|}{ Osteosarcoma } \\
\hline Src activation & {$[71,72]$} \\
\hline Activation of PI3K/Akt signaling pathway & {$[72]$} \\
\hline Caveolin-1 downregulation & [72] \\
\hline c-Met activation & {$[72]$} \\
\hline Ezrin $/ \beta_{4}$ integrin interaction & {$[67]$} \\
\hline $\begin{array}{l}\text { Abbreviations: EFT, Ewing family of tumors } \\
\text { insulin-like growth factor-1 receptor; PI3K, } \\
\text { phosphoinositide 3-kinase. }\end{array}$ & \\
\hline
\end{tabular}

of cell-cell adhesion and the downstream signaling mechanisms dependent on this interaction in anoikis resistance. Using a panel of inhibitory antibodies, anchorage-independent EFT cultures showed a particular dependence on Ecadherin-mediated cell-cell adhesion for survival, as compared with other cadherins and integrins, and this survival was mediated through downstream activation of Akt. Most interestingly, ErbB-4, among a panel of tyrosine kinases using an antibody-based array, was identified as being hyperactivated under detachment conditions, and it was subsequently shown to be critical for anoikis resistance in these EFT cells. The ErbB-4 hyperactivation was E-cadherin dependent, and the E-cadherin-mediated Akt activation was shown to be dependent on ErbB-4 expression. These results are consistent with recent reports showing a protumorigenic role for ErbB-4 in various cancer types. A recent study showed the importance of ErbB-4 in the survival of melanoma cells, specifically in relation to activating ErbB-4 point mutations, which are found in approximately $20 \%$ of melanoma patients [55]. Furthermore, in peripheral nerve sheath tumor cells, neuregulin, a ligand for ErbB receptors, was found to increase migration and invasion by binding to ErbB-3 and ErbB-4 [56]. Kang et al. [54] also demonstrated that anchorage-independent EFT cells were significantly more resistant to chemotherapy than those grown in monolayers and that downregula- 
tion of ErbB-4 restored sensitivity to multiple cytotoxic agents. These data raise the possibility that inhibition of ErbB-4 may serve as a therapeutic target specifically aimed at a stage when the cells are in a micrometastasis-like state, particularly if targeted in combination with chemotherapy. Further in vitro and clinical studies are needed to define the role of ErbB-4 in the metastasis of EFTs, including the specific mechanisms by which ErbB-4 becomes hyperactivated in anchorage-independent conditions, and the possible presence of activating ErbB-4 mutations in primary tumor cases that may further promote its activity. In vivo metastasis studies, including the use of specific kinase inhibitors, are also needed to further support the notion of ErbB-4 as a therapeutic target in this context.

The above results in EFTs also suggest a possible role for cadherin-mediated ligand-independent activation of tyrosine kinases in the context of metastasis. Such a mechanism of action was shown in a study of anoikis resistance in squamous cell carcinoma cells, in which E-cadherin was demonstrated to have a positive role in survival under detachment conditions through its ligand-independent activation of epidermal growth factor receptor [57]. In a colon cancer model, anchorage-independent growth was also found to be dependent on induction of E-cadherin; furthermore, in ovarian surface epithelial cells, ectopically expressed E-cadherin suppressed anoikis and enabled the formation of adenocarcinomas in immunodeficient mice $[58,59]$

There is recent evidence illustrating the importance of factors in anoikis resistance that are not directly linked to modifying the apoptotic program. Specifically, metabolic alterations may play a critical role, and the ability to mitigate the effects of these metabolic changes may be important in cancer progression and resistance to anoikis. A recent report investigated the metabolic status of cells under loss of attachment conditions, using mammary cells as a model system [60]. There was a significant decline in the bioenergetic status of cells as determined by ATP levels following detachment, which could be rescued by expression of a dominant oncogene such as ErbB-2. This drop in ATP levels correlated with decreased glucose uptake, which was also restored in the presence of the ErbB-2 oncogene [59]. Interestingly, reactive oxygen species (ROS) appear to play an important role in the reduction of ATP and glucose uptake levels in detached cells through the ability to suppress fatty acid oxidation, and oncogenic rescue of this bioenergetic stress is in part mediated through its ability to suppress ROS as a result of cellular detachment [59]. Importantly, because of the anoikis-promoting properties of ROS, antioxidants showed protumorigenic effects in the context of anchorage-independent survival, because it promoted both luminal filling in a three-dimensional cell culture model of breast acinar formation and colony formation in soft agar assays. One possible mechanism to mitigate this bioenergetic stress is autophagy, a process of "self-eating" whereby subcellular organelles are broken down through a lysosomal pathway, and component biomolecules are recovered for catabolic processes. A recent study demonstrated autophagy as an important factor in anoikis resistance, because breast epithelial cells deficient in various components of the autophagy pathway showed greater susceptibility to anoikis [61]. Overall, these studies provide new evidence to suggest that resistance to anoikis is, in part, mediated by the ability of cells to tolerate the bioenergetic stress that is the consequence of the loss of cellular attachment. This adds a new branch to our understanding of anoikis resistance, one that functions independently of the status of the apoptotic machinery. Preliminary data suggest that similar mechanisms may be operating in EFTs and are the subject of ongoing investigation.

\section{ANOIKIS Resistance In OSTEOSARComa}

In osteosarcoma, the molecular basis underlying the regulation of micrometastatic disease is very poorly understood, although several of investigations have provided new insights into the development of metastases and resistance to anoikis (Table 1). Ezrin, a membrane-cytoskeletal linker protein, was demonstrated to be an important regulator of the metastatic behavior of osteosarcoma [62, 63]. Khanna and colleagues demonstrated that ezrin was required for the development of metastases in a mouse model and, in dogs and patients with osteosarcoma, high expression levels correlated with poor outcome [63]. Ezrin's ability to link the cell membrane to the actin cytoskeleton allows cells to interact directly with its microenvironment, thereby facilitating signal transduction through growth factor receptors and adhesion molecules $[64,65]$. In a mouse model, ezrinmediated survival of metastases was found to be partially dependent on MAPK signaling, and also dependent on activation of mammalian target of rapamycin, because inhibition with rapamycin inhibited this metastatic behavior $[63,66]$. More recently, the same group demonstrated that expression of a cell adhesion receptor, $\beta_{4}$ integrin, appears to promote metastasis in osteosarcoma. This protein may be important in the survival of osteosarcoma cells in the circulation, because knockdown of $\beta_{4}$ integrin in MNNG-HOS cells in vitro prevented cells from growing in anchorageindependent conditions without affecting proliferation in adherent conditions [67]. In vivo knockdown of $\beta_{4}$ integrin in a mouse model suppressed pulmonary metastases, an effect that was dependent upon interaction with ezrin to main- 
tain its expression levels. What is not currently known is how ezrin regulates $\beta_{4}$ integrin.

Although integrins are well known to play a critical role in the survival of normal adherent cells by activating signal transduction pathways, cell-cell contacts are able to activate integrin signaling in anchorage-independent conditions, and cells may avoid anoikis by changing the pattern of integrin expression (reviewed in [68]). This was demonstrated in squamous cell carcinoma cells, in which a switch from $\alpha_{\mathrm{V}} \beta_{5}$ integrin to $\alpha_{\mathrm{V}} \beta_{6}$ integrin protected cells from anoikis, again by activation of PI3K/Akt signaling [69]. Integrin expression was also shown to increase sensitivity to anoikis, such as was found in the osteosarcoma cell line Saos-2, in which overexpression of $\alpha_{4}$ integrin led to an increase in anoikis [70]. This cell line was partially sensitive to detachment-induced cell death, but when made resistant to anoikis, these cells were dependent on Src kinase activation for survival, because pharmacological inhibition of its activity restored anoikis sensitivity. Dephosphorylation of FAK was not found to be essential for anoikis resistance, but the PI3K/Akt pathway was critical for cell survival, with pharmacological inhibition of PI3K activity restoring sensitivity to anoikis-resistant cells [71]. Further evidence of the role of Src in mediating anoikis resistance in osteosarcoma came from Cantiani et al. [72], who found that overexpression of a signaling modulator protein, caveolin-1, in osteosarcoma cells in vitro inhibited anchorageindependent growth by inhibiting Src and c-Met tyrosine kinases. Caveolin-1 is a protein required for forming plasma membrane invaginations called caveolae that regulate several intracellular signaling pathways [73]. In the above experiments, a panel of osteosarcoma cell lines was used, and Saos-2 cells, which are most sensitive to anoikis, displayed higher basal levels of caveolin-1 than other cells lines. Abrogation of caveolin-1 in Saos- 2 cells using an antisense strategy resulted in greater resistance to anoikis, with greater Src kinase and Met activity. Its role in modulating metastasis was further demonstrated in vitro, whereby abrogation of caveolin-1 increased migration and invasion of osteosarcoma cells, and in vivo, where abrogation dramatically enhanced lung metastasis in athymic mice [73]. The role of caveolin-1 appears to depend upon its cellular context, and it has been described as an oncogene or a tumor-suppressor gene depending on the cell type [74]. Caveolin has been found to suppress anoikis in malignant cell lines, including breast cancer and other mesenchymal tumors; however, in prostate and lung cancer it has a protumorogenic role, and in EFTs it promotes both anchorageindependent and tumor growth in human and mouse models [75-79]. Caveolin-1 has also been found to mediate anoikis through regulation of integrin control of several growth- regulatory pathways, thus potentially providing an additional level to the regulation of anoikis [80].

Src kinase activity has been implicated as a prometastatic pathway in a variety of tumor types because it promotes key oncogenic mechanisms such as cell proliferation, adhesion, apoptotic resistance, and invasion [81]. Initial investigation in osteosarcoma supported this hypothesis because inhibition of Src activity with a small molecule inhibitor, dasatinib, was shown to inhibit migration and invasion of osteosarcoma cells in vitro and tumor growth in vivo [82]. More recently, however, the role of Src in mediating metastasis was questioned because Hingorani and colleagues found that dasatinib failed to alter the development of metastases in an in vivo model of osteosarcoma [83]. Metastases formed regardless of whether dasatinib was commenced 2 days prior to injection of tumor cell at the primary site or 7 days thereafter, implying that Src may not be required for any of the steps of the metastatic process in that model. The authors concluded that inhibition of Src kinase may not be as useful a strategy to prevent pulmonary metastases as had been suggested by the in vitro data alone. If, however, inhibition of Src increases the sensitivity of ostesarcoma cells to anoikis, it may continue to have a role in combination with chemotherapy for eradication of micrometastatic disease.

The pathogenic role of the Notch signaling pathway has been investigated in osteosarcoma. Altered Notch signaling has been associated with many different diseases, including cancer, and it is also involved in bone development [8486]. Thus, Zhang and colleagues investigated its importance in the pathogenesis of osteosarcoma and its role in metastasis [87]. Those authors demonstrated that Notch activation promoted invasion in osteosarcoma cell lines and the development of metastases in a mouse model, effects that were eliminated by chemical inhibition of Notch signaling by $\gamma$-secretase. Interestingly, in the in vitro model, $\gamma$-secretase did not affect cell proliferation or anchorageindependent growth [87]. More recently, Notch and related genes were found to be upregulated in human osteosarcoma cell lines and primary human osteosarcoma tumor samples [88]. In those studies, chemical and genetic Notch inhibition decreased osteosarcoma cell proliferation in vitro and decreased tumor growth in vivo [88]. However, the authors were unable to detect any obvious metastasis of the tumors generated in nude mice, and thus were unable to confirm that Notch inhibition had any effect on metastatic development. Further evidence is required before Notch inhibition can be considered as a therapeutic strategy for the prevention of metastatic disease or eradication of micrometastatic disease. These studies highlight the complexity of investigating the molecular mechanisms 
underlying anoikis resistance and the promotion of metastasis. Determining the most critical cell surface molecules and signaling pathways for survival in such conditions among the many possible candidates is paramount in identifying novel targets that may be exploited for therapeutic benefit.

\section{Conclusions ANd Future Studies}

Cell growth in anchorage-independent conditions provides a valuable in vitro model of micrometastatic disease, because resistance to anoikis and survival in the absence of attachment to the ECM is a prerequisite for the successful development of metastases. The growth characteristics of these cells differ from those of cells grown in adherent conditions because nonadherent cells have lower proliferation rates and they demonstrate greater resistance to chemotherapy. This may, in part, explain why current chemotherapy protocols are unable to eradicate micrometastatic disease in a significant proportion of patients with EFTs and osteosarcoma, and why metastases develop late in the course of the disease in some patients. Activation of certain downstream signaling pathways has consistently been found to be important in anoikis resistance, including PI3K/Akt in EFTs, osteosarcoma, and many other malignancies, and Src signaling in osteosarcoma. Constitutive activation of receptor tyrosine kinases, such as IGF-1R in EFTs, may be important in anchorage-independent cell survival, but cell-cell contact and activation of cadherin and integrins also appear to be required for survival through the formation of multicellular spheroids. These may allow for the ligand-independent activation of novel receptor tyrosine kinases, such as ErbB-4, as was demonstrated in EFTs. Regulation of ErbB-4 under nonadherent growth conditions is currently being investigated, as is the feasibility of inhibiting ErbB-4 as a novel therapeutic target. Anoikis resistance has been less well studied in osteosarcoma, and many aspects of its regulation are not understood, particularly with respect to which proteins and kinases are most critical for cell survival in nonadherent cells. Loss-of-function high-throughput screening strategies may be useful for identifying these and other potential new targets for therapeutic intervention. There are data emerging that metabolic regulation of detached cells and autophagy may contribute to anoikis resistance in epithelial cells. What is not known is whether such processes are also involved in the suppression of anoikis in sarcomas, and this is also the subject of ongoing investigations. In conclusion, it is hoped that a better understanding of micrometastatic disease and anoikis resistance will lead to the identification of novel agents that may be used to eradicate CTCs and reduce the development of metastatic disease in patients with EFTs and osteosarcoma.

\section{ACKNOWLEDGMENTS}

This work was supported by: UCLH/UCL Comprehensive Biomedical Research Centre and Sarcoma Research through Collaboration (SARC) (S.J.S); Canadian Institutes of Health Research (CIHR), Michael Smith Foundation for Health Research (MSFHR), and the Royal College of Physicians of Canada Clinician Investigator Program (T.N.); Children with Leukaemia (A.M.); UCLH/UCL Comprehensive Biomedical Research Centre (J.W.); National Cancer Institute of Canada, CIHR, Department of Defence (DoD), and National Institutes of Health (NIH) (P.H.B.S).

\section{Author Contributions}

Conception/Design: Sandra J. Strauss, Ariadna Mendoza-Naranjo, Jeremy Whelan, Poul H.B. Sorensen, Tony Ng

Manuscript writing: Sandra J. Strauss, Ariadna Mendoza-Naranjo, Jeremy Whelan, Poul H.B. Sorensen, Tony Ng

Final approval of manuscript: Sandra J. Strauss, Ariadna Mendoza-Naranjo, Jeremy Whelan, Poul H.B. Sorensen, Tony Ng

\section{REFERENCES}

1 Sporn MB. The war on cancer. Lancet 1996;347:1377-1381.

2 Riethdorf S, Wikman H, Pantel K. Review: Biological relevance of disseminated tumor cells in cancer patients. Int J Cancer 2008;123:1991-2006.

3 Braun S, Vogl FD, Naume B et al. A pooled analysis of bone marrow micrometastasis in breast cancer. N Engl J Med 2005;353:793-802.

4 Braun S, Kentenich C, Janni W et al. Lack of effect of adjuvant chemotherapy on the elimination of single dormant tumor cells in bone marrow of high-risk breast cancer patients. J Clin Oncol 2000;18:80-86.

5 Janni W, Gastroph S, Hepp F et al. Prognostic significance of an increased number of micrometastatic tumor cells in the bone marrow of patients with first recurrence of breast carcinoma. Cancer 2000;88:2252-2259.

6 Pachmann K, Camara O, Kavallaris A et al. Monitoring the response of circulating epithelial tumor cells to adjuvant chemotherapy in breast cancer allows detection of patients at risk of early relapse. J Clin Oncol 2008;26: $1208-1215$.
7 Mïler V, Stahmann N, Riethdorf S et al. Circulating tumor cells in breast cancer: Correlation to bone marrow micrometastases, heterogeneous response to systemic therapy and low proliferative activity. Clin Cancer Res 2005;11:3678-3685.

8 Goss P, Allan AL, Rodenhiser DI et al. New clinical and experimental approaches for studying tumor dormancy: Does tumor dormancy offer a therapeutic target? APMIS 2008;116:552-568.

9 Link MP. Adjuvant therapy in the treatment of osteosarcoma. Important Adv Oncol 1986:193-207.

10 Meyers PA, Heller G, Healey J et al. Chemotherapy for nonmetastatic osteogenic sarcoma: The Memorial Sloan-Kettering experience. J Clin Oncol 1992;10:5-15.

11 Souhami RL, Craft AW, Van der Eijken JW et al. Randomised trial of two regimens of chemotherapy in operable osteosarcoma: A study of the European Osteosarcoma Intergroup. Lancet 1997;350:911-917.

12 Bruland OS, Hoifodt H, Saeter G et al. Hematogenous micrometastases in osteosarcoma patients. Clin Cancer Res 2005;11:4666-4673. 
13 Arvand A, Denny CT. Biology of EWS/ETS fusions in Ewing's family tumors. Oncogene 2001;20:5747-5754.

14 Delattre O, Zucman J, Plougastel B et al. Gene fusion with an ETS DNAbinding domain caused by chromosome translocation in human tumours. Nature 1992;359:162-165.

15 Sorensen PH, Lessnick SL, Lopez-Terrada D et al. A second Ewing's sarcoma translocation, $\mathrm{t}(21 ; 22)$, fuses the EWS gene to another ETS-family transcription factor, ERG. Nat Genet 1994;6:146-151.

16 Grier HE, Krailo MD, Tarbell NJ et al. Addition of ifosfamide and etoposide to standard chemotherapy for Ewing's sarcoma and primitive neuroectodermal tumor of bone. N Engl J Med 2003;348:694-701.

17 Paulussen M, Craft AW, Lewis I et al. Results of the EICESS-92 Study: Two randomized trials of Ewing sarcoma treatment--cyclophosphamide compared with ifosfamide in standard-risk patients and assessment of benefit of etoposide added to standard treatment in high-risk patients. J Clin Oncol 2008;26:4385-4393.

18 Cotterill SJ, Ahrens S, Paulussen M et al. Prognostic factors in Ewing's tumor of bone: Analysis of 975 patients from the European Intergroup Cooperative Ewing's Sarcoma Study Group. J Clin Oncol 2000;18:3108-3114.

19 Schleiermacher G, Peter M, Oberlin O et al. Increased risk of systemic relapses associated with bone marrow micrometastasis and circulating tumor cells in localized Ewing tumor. J Clin Oncol 2003;21:85-91.

20 Evdokimova V, Tognon C, Ng T et al. Translational activation of Snail1 and other developmentally regulated transcription factors by YB-1 promotes an epithelial-mesenchymal transition. Cancer Cell 2009;15:402415 .

21 Nguyen DX, Bos PD, Massagué J. Metastasis: From dissemination to organ-specific colonization. Nat Rev Cancer 2009;9:274-284.

22 Nguyen DX, Massagué J. Genetic determinants of cancer metastasis. Nat Rev Genet 2007;8:341-352.

23 Frisch SM, Screaton RA. Anoikis mechanisms. Curr Opin Cell Biol 2001; 13:555-562.

24 Frisch SM, Francis H. Disruption of epithelial cell-matrix interactions induces apoptosis. J Cell Biol 1994;124:619-626.

25 Gilmore AP. Anoikis. Cell Death Differ 2005;12(suppl 2):1473-1477.

26 Grossmann J, Walther K, Artinger M et al. Apoptotic signaling during initiation of detachment-induced apoptosis ("anoikis") of primary human intestinal epithelial cells. Cell Growth Differ 2001;12:147-155.

27 Boudreau N, Sympson CJ, Werb Z et al. Suppression of ICE and apoptosis in mammary epithelial cells by extracellular matrix. Science 1995;267: 891-893.

28 Meredith JE Jr, Fazeli B, Schwartz MA. The extracellular matrix as a cell survival factor. Mol Biol Cell 1993;4:953-961.

29 Yawata A, Adachi M, Okuda $\mathrm{H}$ et al. Prolonged cell survival enhances peritoneal dissemination of gastric cancer cells. Oncogene 1998;16:26812686.

30 van Nimwegen MJ, van de Water B. Focal adhesion kinase: A potential target in cancer therapy. Biochem Pharmacol 2007;73:597-609.

31 Shaw LM, Rabinovitz I, Wang HH et al. Activation of phosphoinositide 3-OH kinase by the $\alpha_{6} \beta_{4}$ integrin promotes carcinoma invasion. Cell 1997; 91:949-960.

32 Dufour G, Demers MJ, Gagné D et al. Human intestinal epithelial cell survival and anoikis. Differentiation state-distinct regulation and roles of protein kinase B/Akt isoforms. J Biol Chem 2004;279:44113-44122.

33 Douma S, Van Laar T, Zevenhoven J et al. Suppression of anoikis and induction of metastasis by the neurotrophic receptor TrkB. Nature 2004;430: $1034-1039$
34 Cheng H, Liu P, Wang ZC et al. SIK1 couples LKB1 to p53-dependent anoikis and suppresses metastasis. Sci Signal 2009:2:ra35.

35 Windham TC, Parikh NU, Siwak DR et al. Src activation regulates anoikis in human colon tumor cell lines. Oncogene 2002;21:7797-7807.

36 Wei L, Yang Y, Zhang X et al. Altered regulation of Src upon cell detachment protects human lung adenocarcinoma cells from anoikis. Oncogene 2004;23:9052-9061.

37 Attwell S, Roskelley C, Dedhar S. The integrin-linked kinase (ILK) suppresses anoikis. Oncogene 2000;19:3811-3815.

38 Collins NL, Reginato MJ, Paulus JK et al. G1/S cell cycle arrest provides anoikis resistance through Erk-mediated Bim suppression. Mol Cell Biol 2005;25:5282-5291.

39 Lagadec C, Meignan S, Adriaenssens E et al. TrkA overexpression enhances growth and metastasis of breast cancer cells. Oncogene 2009;28: 1960-1970.

40 Zong CS, Zeng L, Jiang Y et al. Stat3 plays an important role in oncogenic Ros- and insulin-like growth factor I receptor-induced anchorage-independent growth. J Biol Chem 1998;273:28065-28072.

41 Cheng TL, Symons M, Jou TS. Regulation of anoikis by Cdc42 and Rac1. Exp Cell Res 2004;295:497-511.

42 Lawlor ER, Scheel C, Irving J et al. Anchorage-independent multi-cellular spheroids as an in vitro model of growth signaling in Ewing tumors. Oncogene 2002;21:307-318.

43 Idogawa M, Adachi M, Minami T et al. Overexpression of BAD preferentially augments anoikis. Int J Cancer 2003;107:215-223.

44 Schmelzle T, Mailleux AA, Overholtzer M et al. Functional role and oncogene-regulated expression of the BH3-only factor Bmf in mammary epithelial anoikis and morphogenesis. Proc Natl Acad Sci U S A 2007;104: 3787-3792.

45 Woods NT, Yamaguchi H, Lee FY et al. Anoikis, initiated by Mcl-1 degradation and Bim induction, is deregulated during oncogenesis. Cancer Res 2007;67:10744-10752.

46 Hofbauer S, Hamilton G, Theyer G et al. Insulin-like growth factor-Idependent growth and in vitro chemosensitivity of Ewing's sarcoma and peripheral primitive neuroectodermal tumour cell lines. Eur J Cancer 1993; 29A:241-245

47 Scotlandi K, Benini S, Sarti M et al. Insulin-like growth factor I receptormediated circuit in Ewing's sarcoma/peripheral neuroectodermal tumor: A possible therapeutic target. Cancer Res 1996;56:4570-4574.

48 Toretsky JA, Kalebic T, Blakesley V et al. The insulin-like growth factor-I receptor is required for EWS/FLI-1 transformation of fibroblasts. J Biol Chem 1997;272:30822-30827.

49 Martin MJ, Melnyk N, Pollard M et al. The insulin-like growth factor I receptor is required for Akt activation and suppression of anoikis in cells transformed by the ETV6-NTRK3 chimeric tyrosine kinase. Mol Cell Biol 2006;26:1754-1769.

50 Scotlandi K, Maini C, Manara MC et al. Effectiveness of insulin-like growth factor I receptor antisense strategy against Ewing's sarcoma cells. Cancer Gene Ther 2002;9:296-307.

51 Valentinis B, Reiss K, Baserga R. Insulin-like growth factor-I-mediated survival from anoikis: Role of cell aggregation and focal adhesion kinase. J Cell Physiol 1998;176:648-657.

52 Kim SY, Wan X, Helman LJ. Targeting IGF-1R in the treatment of sarcomas: Past, present and future. Bull Cancer 2009;96:E52-E60.

53 Manara MC, Landuzzi L, Nanni P et al. Preclinical in vivo study of new insulin-like growth factor-I receptor-specific inhibitor in Ewing's sarcoma. Clin Cancer Res 2007;13:1322-1330. 
54 Kang HG, Jenabi JM, Zhang J et al. E-cadherin cell-cell adhesion in Ewing tumor cells mediates suppression of anoikis through activation of the ErbB4 tyrosine kinase. Cancer Res 2007;67:3094-3105.

55 Prickett TD, Agrawal NS, Wei X et al. Analysis of the tyrosine kinome in melanoma reveals recurrent mutations in ERBB4. Nat Genet 2009;41: $1127-1132$.

56 Eckert JM, Byer SJ, Clodfelder-Miller BJ et al. Neuregulin-1 beta and neuregulin-1 alpha differentially affect the migration and invasion of malignant peripheral nerve sheath tumor cells. Glia 2009;57:1501-1520.

57 Shen X, Kramer RH. Adhesion-mediated squamous cell carcinoma survival through ligand-independent activation of epidermal growth factor receptor. Am J Pathol 2004;165:1315-1329.

58 Mueller S, Cadenas E, Schönthal AH. p21WAF1 regulates anchorageindependent growth of HCT116 colon carcinoma cells via E-cadherin expression. Cancer Res 2000;60:156-163.

59 Ong A, Maines-Bandiera SL, Roskelley CD et al. An ovarian adenocarcinoma line derived from SV40/E-cadherin-transfected normal human ovarian surface epithelium. Int J Cancer 2000;85:430-437.

60 Schafer ZT, Grassian AR, Song L et al. Antioxidant and oncogene rescue of metabolic defects caused by loss of matrix attachment. Nature 2009;461: 109-113.

61 Fung C, Lock R, Gao S et al. Induction of autophagy during extracellular matrix detachment promotes cell survival. Mol Biol Cell 2008;19:797806.

62 Algrain M, Turunen O, Vaheri A et al. Ezrin contains cytoskeleton and membrane binding domains accounting for its proposed role as a membrane-cytoskeletal linker. J Cell Biol 1993;120:129-139.

63 Khanna C, Wan X, Bose S et al. The membrane-cytoskeleton linker ezrin is necessary for osteosarcoma metastasis. Nat Med 2004;10:182-186.

64 Arpin M, Algrain M, Louvard D. Membrane-actin microfilament connections: An increasing diversity of players related to band 4.1. Curr Opin Cell Biol 1994;6:136-141.

65 Fazioli F, Wong WT, Ullrich SJ et al. The ezrin-like family of tyrosine kinase substrates: Receptor-specific pattern of tyrosine phosphorylation and relationship to malignant transformation. Oncogene 1993;8:1335-1345.

66 Wan X, Mendoza A, Khanna C et al. Rapamycin inhibits ezrin-mediated metastatic behavior in a murine model of osteosarcoma. Cancer Res 2005; 65:2406-2411.

67 Wan X, Kim SY, Guenther LM et al. $\beta_{4}$ integrin promotes osteosarcoma metastasis and interacts with ezrin. Oncogene 2009;28:3401-3411.

68 Chiarugi P, Giannoni E. Anoikis: A necessary death program for anchorage-dependent cells. Biochem Pharmacol 2008;76:1352-1364.

69 Janes SM, Watt FM. Switch from $\alpha_{\mathrm{v}} \beta_{5}$ to $\alpha_{\mathrm{v}} \beta_{6}$ integrin expression protects squamous cell carcinomas from anoikis. J Cell Biol 2004;166:419-431.

70 Marco RA, Díaz-Montero CM, Wygant JN et al. $\alpha_{4}$ integrin increases anoikis of human osteosarcoma cells. J Cell Biochem 2003;88:1038-1047.

71 Diaz-Montero CM, Wygant JN, McIntyre BW. PI3-K/Akt-mediated anoikis resistance of human osteosarcoma cells requires Src activation. Eur J Cancer 2006;42:1491-1500.
72 Cantiani L, Manara MC, Zucchini C et al. Caveolin-1 reduces osteosarcoma metastases by inhibiting c-Src activity and met signaling. Cancer Res 2007;67:7675-7685.

73 Rothberg KG, Heuser JE, Donzell WC et al. Caveolin, a protein component of caveolae membrane coats. Cell 1992;68:673-682.

74 Goetz JG, Lajoie P, Wiseman SM et al. Caveolin-1 in tumor progression: The good, the bad and the ugly. Cancer Metastasis Rev 2008;27:715-735.

75 Fiucci G, Ravid D, Reich R et al. Caveolin-1 inhibits anchorage-independent growth, anoikis and invasiveness in MCF-7 human breast cancer cells. Oncogene 2002;21:2365-2375.

76 Wiechen K, Sers C, Agoulnik A et al. Down-regulation of caveolin-1, a candidate tumor suppressor gene, in sarcomas. Am J Pathol 2001;158:833839.

77 Bangma CH, Nasu Y, Ren C et al. Metastasis-related genes in prostate cancer. Semin Oncol 1999;26:422-427.

78 Ho CC, Huang PH, Huang HY et al. Up-regulated caveolin-1 accentuates the metastasis capability of lung adenocarcinoma by inducing filopodia formation. Am J Pathol 2002;161:1647-1656.

79 Tirado OM, Mateo-Lozano S, Villar J et al. Caveolin-1 (CAV1) is a target of EWS/FLI-1 and a key determinant of the oncogenic phenotype and tumorigenicity of Ewing's sarcoma cells. Cancer Res 2006;66:9937-9947.

80 Zhang H, Su L, Mïler S et al. Restoration of caveolin-1 expression suppresses growth and metastasis of head and neck squamous cell carcinoma. Br J Cancer 2008;99:1684-1694.

81 Summy JM, Gallick GE. Src family kinases in tumor progression and metastasis. Cancer Metastasis Rev 2003;22:337-358.

82 Shor AC, Keschman EA, Lee FY et al. Dasatinib inhibits migration and invasion in diverse human sarcoma cell lines and induces apoptosis in bone sarcoma cells dependent on SRC kinase for survival. Cancer Res 2007;67: $2800-2808$

83 Hingorani P, Zhang W, Gorlick R et al. Inhibition of Src phosphorylation alters metastatic potential of osteosarcoma in vitro but not in vivo. Clin Cancer Res 2009;15:3416-3422.

84 Bolós V, Grego-Bessa J, de la Pompa JL. Notch signaling in development and cancer. Endocr Rev 2007;28:339-363.

85 Engin F, Yao Z, Yang T et al. Dimorphic effects of Notch signaling in bone homeostasis. Nat Med 2008;14:299-305.

86 Hilton MJ, Tu X, Wu X et al. Notch signaling maintains bone marrow mesenchymal progenitors by suppressing osteoblast differentiation. Nat Med 2008; 14:306-314.

87 Zhang P, Yang Y, Zweidler-McKay PA et al. Critical role of notch signaling in osteosarcoma invasion and metastasis. Clin Cancer Res 2008;14: 2962-2969.

88 Engin F, Bertin T, Ma O et al. Notch signaling contributes to the pathogenesis of human osteosarcomas. Hum Mol Genet 2009;18:1464-1470.

89 Simpson CD, Anyiwe K, Schimmer AD. Anoikis resistance and tumor metastasis. Cancer Lett 2008;272:177-185. 